\title{
SMART ChaIR: IoT BASEd HEALTH TELEMETRY FOR AIR TRAFFIC CONTROLLERS AND EMERGENCY RESPONSE CENTER OPERATORS
}

\author{
Nodirkhon Yusupov, Sergii Akhinko, Rohin Sambath Kumar, \\ Harish Kumar Singh, Rida Khan \& Nikhil Kashyap
}
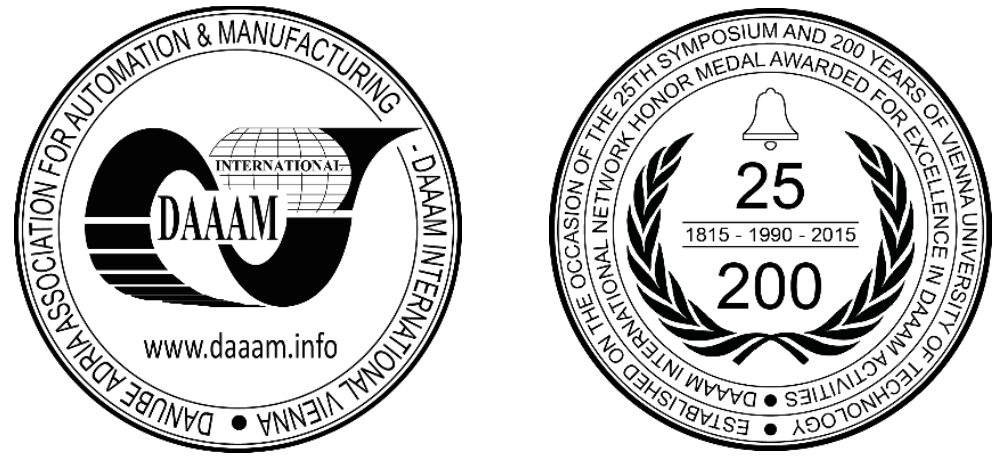

This Publication has to be referred as: Yusupov, N[odirkhon]; Akhinko, S[ergeii]; Sambath Kumar, R[ohin]; Kumar Singh, H[arish]; Khan, R[ida] \& Nikhil, K[ashyap] (2018). Smart Chair: IoT Based Health Telemetry for Air Traffic Controllers and Emergency Response Center Operators, Proceedings of the 29th DAAAM International Symposium, pp.1279-1285, B. Katalinic (Ed.), Published by DAAAM International, ISBN 978-3-902734-20-4, ISSN 1726-9679, Vienna, Austria

DOI: $10.2507 / 29$ th.daaam.proceedings. 184

\begin{abstract}
A correlation exists between decision-making process and physiological as well as psychosocial conditions of air traffic controllers (ATC) and emergency response center operators. The erroneous decisions made by them may lead to accidents that can put human life at risk. A SmartChair system with Internet-of-Things (IoT) based health telemetry is proposed in this paper as a solution to minimize the effects of physiological and psychosocial factors on decision-making process. The given architecture integrates a security system for workers identification and other modules for monitoring their physiological and psychosocial attributes. The first prototype of SmartChair system is implemented and tested. The generated results and observations indicate that the SmartChair system is a feasible and efficient tool for continuous health telemetry.
\end{abstract}

Keywords: ATC; IoT; health monitoring; text-to-speech; speech-to-text; cloud processing

\section{Introduction}

Air traffic controllers (ATC) and operators at emergency response centers are dealing throughout the day and 7 days a week with a large number of crucial situations where the lives of others depend on the decisions made by them. The Estonian air traffic controllers handled 206563 flights with an average of 564 fights per day in 2016 [1]. Moreover, the Estonian regional coordination centers directing fire fighting, ambulance and police services deal with around 1.5 million calls and 2.2 million radio communication contacts per year [2].The work load experienced by such workers may lead to the prevalence of stress, fatigue, disorientation, vertigo, anxiety, panic, mental pressure, overload and task saturation. According to the studies conducted in [3], the most prevalent disorders among ATC workers were concerned with digestive, neuropsychic and cardiovascular systems. This urges the necessity for the continuous monitoring of ATCs physical and mental conditions as well as a continuous improvement of their working situations. 
With the advent of Internet-of-things (IoT) and health telemetry, a more effective and efficient platform can be provided for the continuous supervision of ATCs health and working environment. The usage of SmartChair system, with the reliable security and health monitoring capabilities, is a reasonable solution to this requirement. For example, authors in [4] proposed a SmartChair with features of weight measurement, posture and heart rate monitoring and instantaneous warning generation for wrong posture. Another research conducted in [5] focused on posture monitoring through smartphone application to help the users correct their unbalanced posture. The implemented prototype in [5] used low power Bluetooth communication to transmit data from sensors to gateway.

A SmartChair system with attributes of vital physiological parameters monitoring and feedback generation on Android phone application is suggested in [6]. To the best of our knowledge, no research work has been dedicated in the domain of ATCs health monitoring and the respective feedback generation, along with the required security levels. The proposed SmartChair prototype not only integrates different sensors for physiological parameters monitoring but also combines feedback mechanism for warning generation, in case of emergency or intrusion. A security module is also included to ensure the usage of SmartChair system by the valid user. In addition, the modular nature of our proposed SmartChair architecture ensures its fast and easy deployment. The remainder of the paper is organized as follows: Section 2 describes the SmartChair system architecture and section 3 explains the system design and its implementation. In Section 4, a discussion on testing and results is provided whereas section 5 delivers conclusion on results.

\section{System architecture}

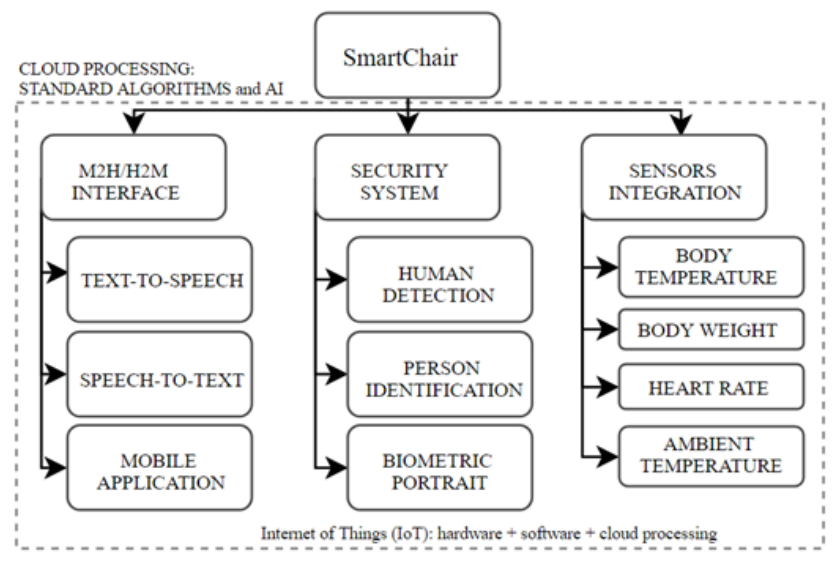

Fig. 1. The SmartChair and its subsystems as donors and users of cloud processing algorithms: 1) M2H and $\mathrm{H} 2 \mathrm{M}$ interfaces; 2) Security system; 3) Biomedical monitoring.

The basic modules and sub-modules of SmartChair system are shown in Fig. 1. Sensors integration module includes different sensors for health monitoring while security system module involves human detection and person identification. Machine-to-human (M2H) and human-to-machine (H2M) in- terface uses mobile application and text-to-speech/speechto-text sub-modules for voice input and warning generation. Human detection is included because someone may try to change the data being collected by introducing a dummy object on the SmartChair. The collected data must be saved at a central location such as remote server for cloud processing and be accessed by the supervisor from own portable devices via mobile application. Various sensors and hardware components utilized in SmartChair system are summarized in Table 1 .

\begin{tabular}{|c|c|}
\hline Requirement & Hardware Component \\
\hline Person Identification & PN-532 RFID Card with Reader \\
\hline Human Detection & S-type Load Cell (HX711 ADC) \\
\hline Stress Monitoring & Pulse Sensor (ADS1015 ADC) \\
\hline Body Temperature Monitoring & IR Thermopile Sensor (TMP006) \\
\hline Room Temperature Monitoring & Ambient Temperature Sensor (DS18B20) \\
\hline Voice Command Generation & USB Microphone \\
\hline Notification and Warning & USB Speaker \\
\hline Control Unit & Raspberry Pi 3 Model B \\
\hline
\end{tabular}

Table 1. Hardware Components for smartchair System Design. 


\section{System design and implementation}

The distribution of modules with subsystems ensures sustainability of the proposed design as modules can be replaced and upgraded. There are four distinctive subsystems, as shown in Fig. 2 and 1. Control unit, having Raspberry Pi, along with speakers and ambient temperature sensor, in a box attached to the strap; 2) Near field communication (NFC) tag mounted on the right handle for person identification; 3) Wristband with microphone (MIC), body temperature and optical pulse sensors; 4) Load cell integrated into soft chair pad.

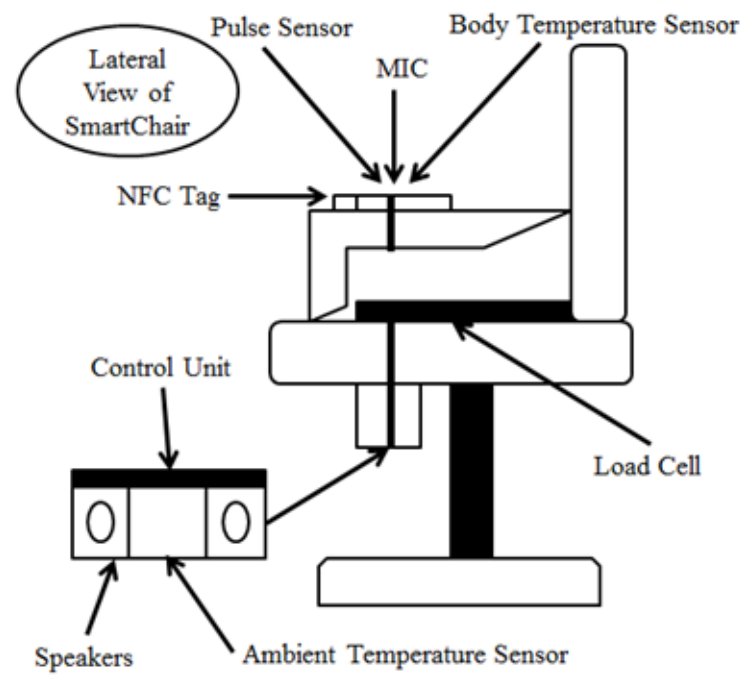

Fig. 2. Lateral view of SmartChair design: 1) Control unit and speakers; 2) Load cell in soft chair pad; 3) Sensors on wristband and MIC; 4) NFC tag.

\subsection{Human Detection and Person Identification}

The ATC must be identified and authorized by the SmartChair system to correctly characterize the collected data. The failure to identify the user will trigger a sound alarm over speakers. Nevertheless, the identification of a user is preceded by human detection procedure. The chair occupancy is detected with a load cell that senses weight of the human body.

Once, it is detected that a chair has been occupied, the user will be greeted and asked to authenticate oneself by placing a personal RFID card on NFC card reader.

Once, the user has used own RFID card for authentication, the unique identification number (UID) will be sent over the Internet to remote server for processing over cloud. If the received UID matches the entry in the database, the server will send confirmation of successful authentication along with the name of the user. Once, the confirmation is received by the SmartChair system, the personified greeting message will be played to the user on portable speakers and the system will commence sending health telemetry data to the server. The entire procedure is depicted in Fig. 3

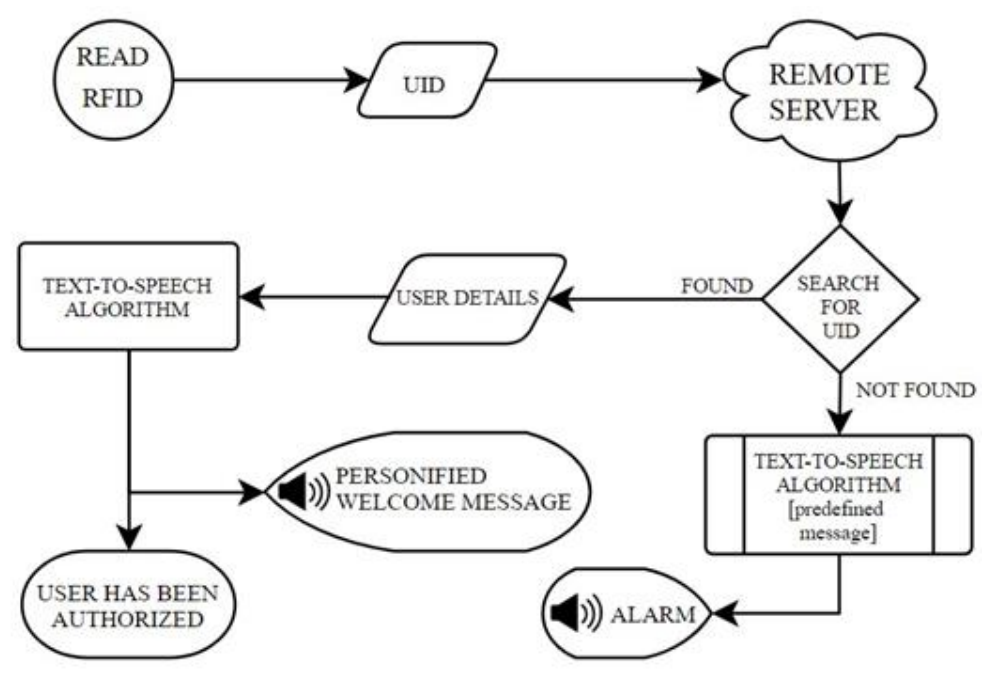

Fig. 3. Person identification algorithm flow-chart along with text-to-speech implementation 


\subsection{Speech-to-Text and Text-to-Speech Recognition}

Generally, the primary form of communication for ATC workers is over voice to avoid distraction. The mentioned above assumptions led to the implementation of speech-to- text and text-to-speech recognition subsystems as solutions for $\mathrm{M} 2 \mathrm{H}$ and $\mathrm{H} 2 \mathrm{M}$ communication. The algorithms for person identification and human detection as well as sensor instrumentation are realized as scripts in Python programming language over Raspberry Pi module. Nevertheless, the text-to- speech and speech-to-text algorithms rely on cloud processing services provided by Google.

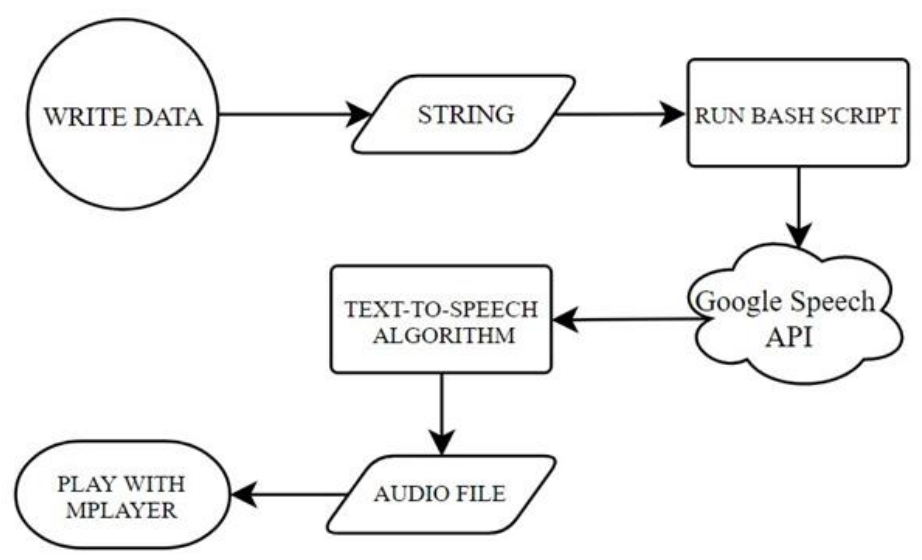

Fig. 4. Text-to-speech algorithm flow chart with core processing done at the remote server side.

Therefore, the data returned from a Python script is sent as a parsed string to a bash script which sends it further to the Google Speech API server. The server processes text in the string and converts it to an audio file that is sent to the SmartChair system where it is played with the Mplayer application, as depicted in Fig. 4. All voice commands are recorded with a microphone. The captured voice commands are sent to the Google Voice Assistant API server where they are converted to text. The converted text is sent back to the SmartChair system and compared against entries in a locally stored configuration file. Once, the match for the keyword has been found, the respective string will be automatically parsed into a bash script and executed, as illustrated in Fig. 5.

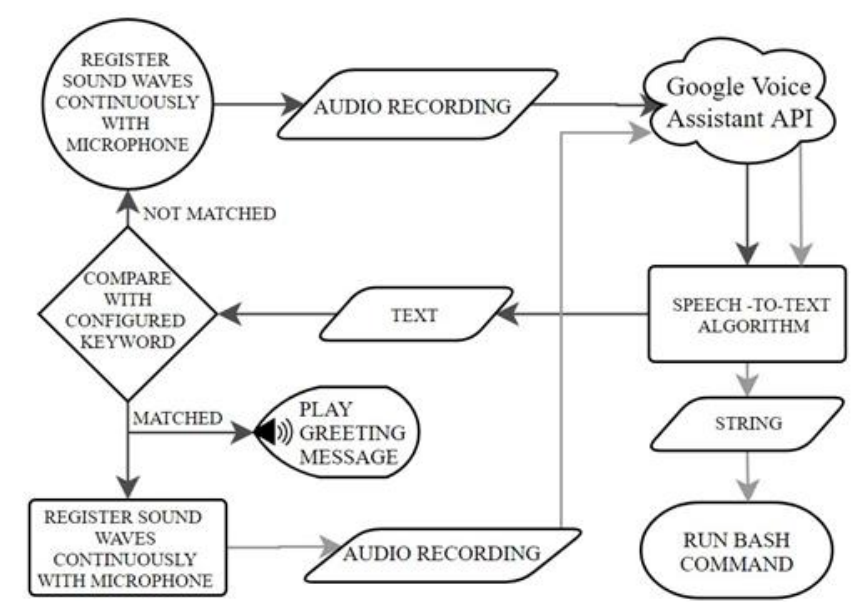

Fig. 5. Speech-to-text algorithm flow chart with core processing done at the remote server side.

\subsection{Sensor Integration and Control Instrumentation}

The physiological parameters of the SmartChair user are obtained with a set of sensors such as load cell, optical pulse sensor and infrared thermopile sensor. The input from load cell is sent to Raspberry Pi computer via analog-to-digital converter (ADC), as mentioned in Table 1. It is noteworthy that the weight of the user is matched against previous results to verify that the right person has been authenticated. While the load cell is placed inside the soft chair pad, optical pulse sensor and infrared thermopile sensor are integrated into a wristband. It was found that these sensors require contact with the skin to produce measurements with a smaller uncertainty range. A separate ADC is used for pulse sensor to interface it with the Raspberry Pi single-board computer. The electromagnetic radiation produced by the human body is absorbed by the infrared thermopile sensor. Finally, the ambient temperature sensor for measuring surrounding temperature is installed along with the single-board Raspberry Pi computer. 


\subsection{Mobile Phone Application}

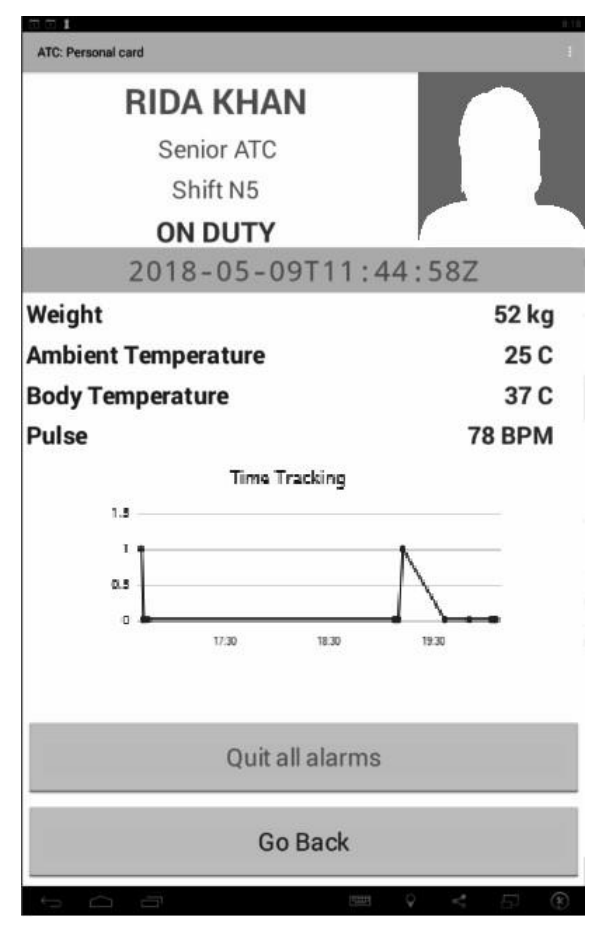

Fig. 6. Information provided by the remote server using processed data obtained from SmartChair and visualized on mobile phone application of supervisor.

The ATC controllers are managed by a shift supervisor who is responsible to ensure the quality of work done by controllers. In order to provide physiological parameter monitoring by supervisor, an Android phone application was developed to provide information based on parameters obtained from the SmartChair. The registered data is sent from SmartChair to the remote server for cloud processing where the data is interpreted, combined, aggregated, and visualized. The mobile phone application sends GET request to the remote server every 2 seconds. The servers reply with a JSON file containing data from the specified channel for the given sensor. The JSON file is processed on mobile phone and obtained data is displayed on the screen with a graphic user interface (GUI), as shown in Fig. 6. The mobile phone application requires the supervisor to authenticate oneself to get authorization to access data related to ATC controllers, as illustrated in Fig. 7. Once the authorization is obtained, the supervisor is able to see the list of employees and all the associated data such as chair occupancy, physiological parameters, and time tracking

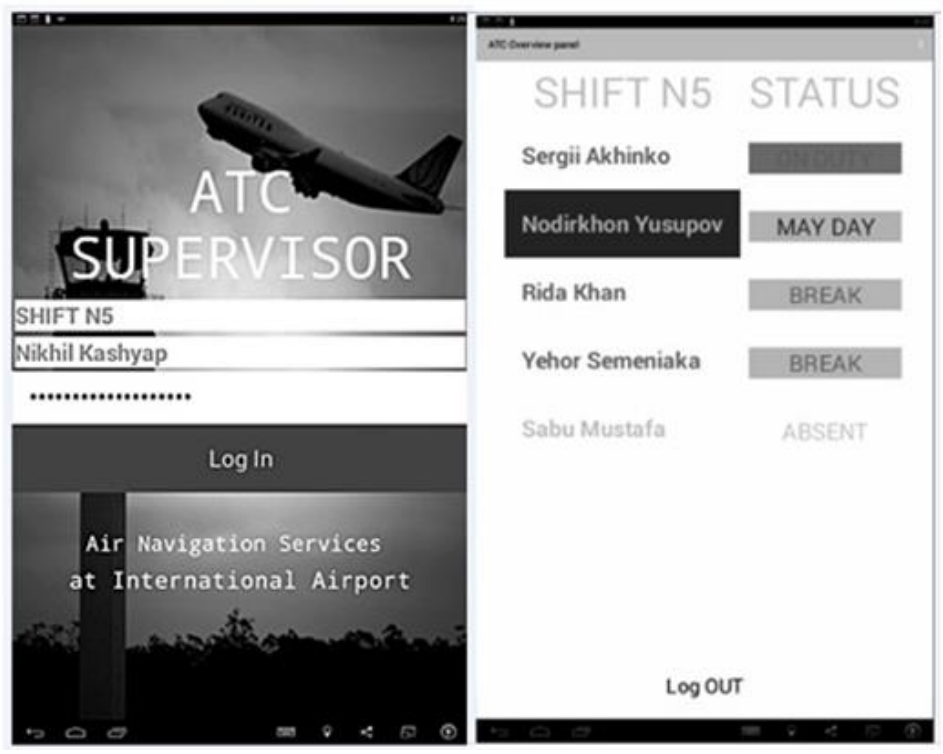

Fig. 7. GUI of mobile phone application for supervisor: Left-Authentication and authorization system; Right-List of employees. 


\section{Testing and results}

To verify the proposed functionality of the SmartChair, the first prototype was built with electric wires connected to wristband (and other devices) and power supplied from the socket with an AC-DC adapter. Also, the data collected from control unit (Raspberry Pi) is sent using the in-built WiFi module via Internet to cloud, as explained in sub-sections 1.1 and 1.2.

The physiological parameters obtained from the SmartChair are body temperature, surrounding temperature, body weight, and pulse rate. The knowledge of body temperature provides some insight into the thermoregulation and metabolism pro- cesses in the ATC body whereas the surrounding temperature helps monitoring the environment favorability. Whenever there is a continuously high temperature for a period of 30 minutes, an audio alarm is triggered on the SmartChair and mobile phone application. The pulse rate measured as beats per minute or cardiac cycle per minute is averaged and the large deviation from the average value for a period of 60 seconds will indicate an increase in stress and will trigger an alarm on SmartChair and the mobile phone of the supervisor.

Several tests were conducted to verify the engineered functionality of subsystems in the working prototype of SmartChair. The aim of the first test was to verify the conve- nience of operation for person identification and human detec- tion subsystems. The volunteer was asked to identify oneself with a personal RFID card via NFC card reader without taking a seat. Nevertheless, the SmartChair successfully determined that chair was not occupied and played an audio message asking the volunteer to take a seat.

Once, the volunteer took seat, the SmartChair played another audio message asking the volunteer to place personal RFID card into the slot located at the right handle. The SmartChair found that UID of the card was not present in the database and triggered an audio alarm on the SmartChair and mobile phone application. Then, the volunteer was asked to use a personal RFID card with UID entered into the database on the remote server and was granted access to SmartChair system after placing the valid RFID card. The aim of the second test was to obtain parameters during relaxed condition. Thus, the volunteer was asked to breathe normally while sitting on the SmartChair. Body temperature, weight and heart rate were measured during the relaxed condition. The data was successfully analyzed at remote server and visualized on GUI of the mobile application, installed on supervisor's phone.

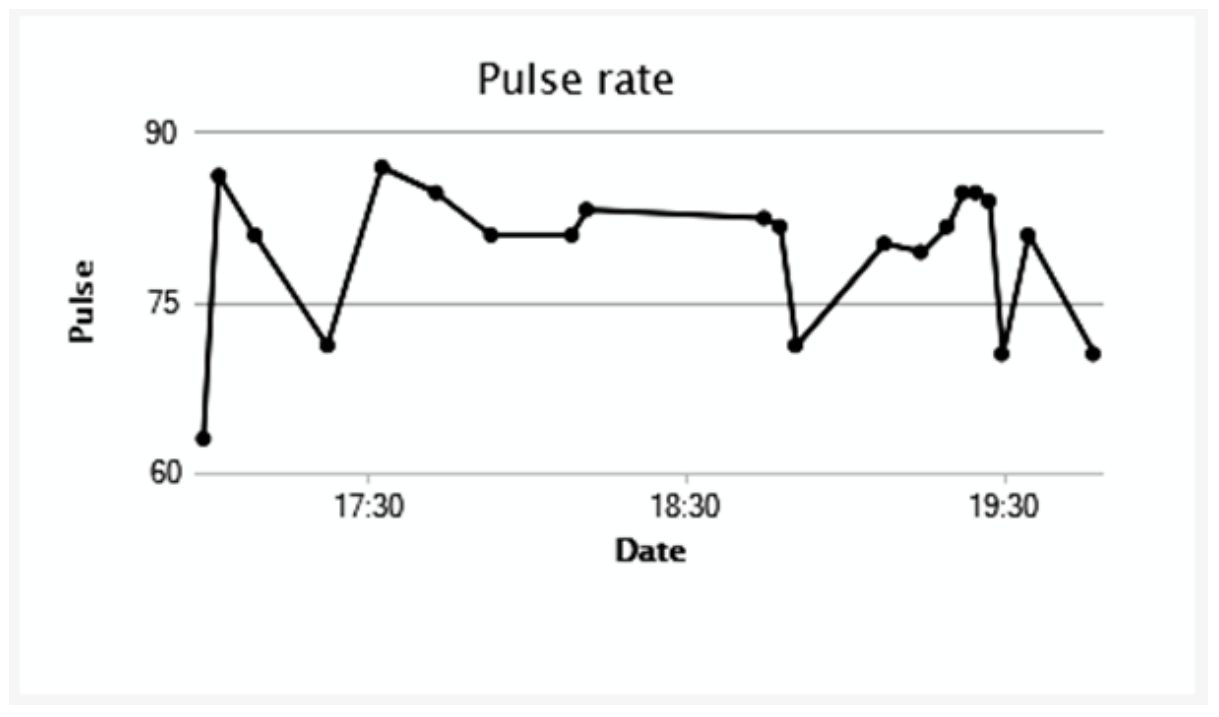

Fig. 8. Plot with data from pulse sensor. Y-axis: Pulse rate in the range from 60 to 90 beats per minute; X-axis: Time lapsed for data registration.

The aim of the third test was to simulate stressful condition and determine its correlation with the pulse rate, as shown in Fig. 8. The volunteer was asked to watch the given horror movie while sitting on the SmartChair. The registered pulse rate showed a significant increase in a number of cardiac cycles during the culmination scenes shown in the horror movie. Therefore, the pulse rate can be used as a primary indicator of psychosocial stress.

\section{Conclusion}

The results obtained after the SmartChair prototype testing were proved to be successful from the health monitoring and the required security viewpoints. Nevertheless, the preliminary stages of SmartChair development require further testing and enhancements to be done. The further steps for the development of this project would be an implementation of an array of sensors such as strain gauge, accelerometers, ECG electrodes and others. Also, the accurate diagnostics would be possible with the accumulation of big data obtained from sensors installed on SmartChair and implementation of machine learning algorithms in cloud processing. 


\section{Acknowledgments}

The authors of this paper would like to express their gratitude to Professor Dr. Mart Tamre and Mr. Andres Eek of the Tallinn University of Technology for their continuous support for the SmartChair project. Special thanks are addressed to Mr. Udayan Sunil Patankar of Sensoramo PLC for reviewing this work. Finally, the authors would like to thank the organizers of the Tallinn Winter School 2018 who provided a basis for the successful development of this project. .

\section{References}

[1] EANS 20, "Your Guide in Estonian Sky! EANS 20," Sept. 2017. [Online]. Available: http://www.eans.ee. [Accessed: April 28, 2018].

[2] European Emergency Number Association, “The Estonian Emergency Response Centre," Sept. 2010. [Online]. Available: http://www.eena.org. [Accessed: April 25, 2018].

[3] G. Costa, "Working and Health Conditions of Italian Air Traffic Controllers," Int. Journal of Occupational Safety and Ergonomics, vol. 6, no. 3, pp. 365-382, 2000.

[4] B. J. Kim, Y. H. Noh, and D. U. Jeong, "Implementation of the Smart Chair System for Multi-functional Unconstrained Healthcare Monitoring," in Proc. 29th Int. Conf. on Advanced Info. Networking and App., Mar. 2015, pp. 448-452.

[5] M. Park, Y. Song, J. Lee, and J. Paek, "Design and Implementation of a Smart Chair System for IoT," in Proc. Int. Conf. on Info. and Comm. Tech. Convergence, Oct. 2016, pp. 1200-1203.

[6] G. R. D. Ganesh, K. Jaidurgamohan, V. Srinu, C. R. Kancharla and S.V. S. Suresh, "Design of a Low Cost Smart Chair for Telemedicine and IoT based Health Monitoring" in Proc. 11th Int. Conf. on Industrial and Info. Systems, Dec. 2016, pp. 89-94. 